\title{
Challenges and Prospective Applications of Extra-oral Implants for Maxillofacial Rehabilitation
}

\section{Sompop Bencharit ${ }^{*}$}

Department of Prosthodontics, School of Dentistry, University of North Carolina at Chapel Hill, Chapel Hill, NC 27599, USA

Since Branemark introduced the concept of osseointegration over three decades ago, dental implants have been widely used to replace teeth and, more importantly, to retain or support intra- and extra-oral prostheses. While intra-oral dental implants are well-developed and thoroughly studied, this is not the case for the extra-oral implants. There are only a handful of longitudinal studies for extra-oral implants. These studies along with several case reports suggest that the success criteria and complications of extra-oral implants are unique to the implant sites. For instance the auricular implants have a survival rate close to $100 \%$. However the success rates were about $75-90 \%$ for the nasal and orbital implants (Abu-Serriah et al. [1], Karakoca et al. [2], Curi MM et al. [3]). Not only does the uniqueness of anatomic structures affect the survival rate of the extra-oral implants, but often times the maxillofacial patients lose anatomical structure from surgical resection and may also undergo chemotherapy or radiation therapy. These pre-prosthetic treatments can further compromise the survival of the implants and increase complications. In addition to this, the extra-oral environment seems to predispose the implants to soft tissue infection that is distinct from the intra-oral implants, where it is rare to have similar soft tissue complications (Arcuri M et al. [4], Abu-Serriah et al. [1]). The improvement of surgical technique, post-op care, and prosthetic fabrication may have improved the implant survival rate and reduced complications (Curi M et al. [3], Goiato et al. [5]).

Recent major advancement in digital dentistry allows significant improvement in the treatment planning for extra-oral implant placement and prothesis fabrication. Cone beam computed tomography (CBCT) provides an accurate measurement of available osseous structure. Furthermore, computer-aided design (CAD) in combination with rapid-prototyping of the surgical guide help surgeons in planning for grafting/reconstructing the implant site and placing implants in a more precise manner, and improve the surgeon's communication with maxillofacial prosthodontists (Van der Meer et al. [6]). This perhaps accounts for the improvement of implant survival rates, especially in the nasal and orbital areas, and the reduction of complications. The digital application also facilitates the fabrication of prostheses.

Because of the larger market share, simplicity of implant placement, and low cost of the components, intra-oral dental implants are widely used, well-developed, and thoroughly studied. Improvement of the designs and applications of intra-oral implants are driven by commercial demands and competition. While there is some recent advancement in extra-oral implants and maxillofacial implantretained/supported prostheses, there is not much development in terms of implant designs and applications, because of limited demand compared to the intra-oral implants/prostheses. The designs and applications of current commercially available extra-oral implants are based solely on the intra-oral ones. In addition to this, the utilization of intra-oral implants is not only driven solely by specialists. Both general dentists and specialists use intra-oral dental implants. While about half of the intra-oral implants are placed by dental specialists, the majority of them are restored by general dentists. Unlike the intra-oral implants, the placement of extra-oral implants require trained oral and maxillofacial or ENT surgeons and most often can only be done in an operating room. The fabrication of the prostheses can only be done by trained prosthodontists or maxillofacial prosthodontists. The extra-oral implant prosthesis is therefore a more complex and costly treatment compared to the intra-oral counterpart.

One of the advantages of extra-oral implant prostheses is that the treatment is often covered by medical insurance in most developed countries. In the US, while the placement of implants in the operating room and in-patient care may be covered by medical insurance, the fabrication of prostheses may not be. Recent debates in the US on the Patient Protection and Affordable Care Act, also known as Health Care Reform that will be enforced in 2014, may have critical effects in helping patients to pay for their maxillofacial prostheses. The US Supreme Court is now reviewing the legality of the Health Care Reform. The court decision will undoubtedly change how patients may or may not be able to afford an implant-retained/supported maxillofacial prosthesis.

In conclusion, we have to carefully apply the knowledge from a limited number of long-term follow up studies of extra-oral implants, and combine this with knowledge from intra-oral and orthopedic implants to further advance the field of extra-oral implant prosthetics. More multi-center based prospective studies with larger populations will be needed to provide sufficient data to examine the effectiveness of implant designs together with the applications of digital technology. We also need to advocate at the government and private sectors for the benefit of filling this gap of knowledge to improve the care for patients with an extra-oral prosthesis.

\section{References}

1. Abu-Serriah MM, McGowan DA, Moos KF, Bagg J (2001) Outcome of extraoral craniofacial endosseous implants. Br J Oral Maxillofac Surg 39: 269-275.

2. Karakoca S, Aydin C, Yilmaz H, Bal BT (2008) Survival rates and periimplant soft tissue evaluation of extraoral implants over a mean follow-up period of three years. J Prosthet Dent 100: 458-464

3. Curi MM, Oliveira MF, Molina G, Cardoso CL, De Groot Oliveira L, et al (2012) Extraoral implants in the rehabilitation of craniofacial defects: implan and prosthesis survival rates and peri-implant soft tissue evaluation. $\mathrm{J}$ Oral Maxillofac Surg 70: 1551-1557.

4. Arcuri M, LaVelle W, Higuchi KW, Hofman HH (1993) Combined percutaneouspermucosal titanium implants for retention of a maxillary prosthesis: a clinical report. J Prosthet Dent 70: 288-290.

*Corresponding Author: Sompop Bencharit, DDS, MS, PhD, FACP, Assistant Professor, Department of Prosthodontics, School of Dentistry, University of North Carolina at Chapel Hill, CB\#7450, Chapel Hill, NC 27599-7450, USA, E-mail: Sompop_Bencharit@dentistry.unc.edu

Received June 23, 2012; Accepted June 23, 2012; Published June 26, 2012

Citation: Bencharit S (2012) Challenges and Prospective Applications of Extra-ora Implants for Maxillofacial Rehabilitation. Anaplastology 1:e103. doi: 10.4172/2161$1173.1000 \mathrm{e} 103$

Copyright: (c) 2012 Bencharit S. This is an open-access article distributed unde the terms of the Creative Commons Attribution License, which permits unrestricted use, distribution, and reproduction in any medium, provided the original author and source are credited. 
Citation: Bencharit S (2012) Challenges and Prospective Applications of Extra-oral Implants for Maxillofacial Rehabilitation. Anaplastology 1:e103. doi: $10.4172 / 2161-1173.1000 \mathrm{e} 103$

Page 2 of 2

5. Goiato MC, Dos Santos DM, Haddad MF, Moreno A (2012) Rehabilitation with ear prosthesis linked to osseointegrated implants. Gerodontology 29: 150-154.
6. Van der Meer WJ, Vissink A, Raghoebar GM, Visser A (2012) Digitally designed surgical guides for placing extraoral implants in the mastoid area. Int $\mathrm{J}$ Oral Maxillofac Implants 27: 703-707. 